\title{
Mesenteric Fibromyoma Mimicking a Colon Tumor: A Diagnostic Dilemma
}

\author{
Safak Ozturk ${ }^{1}$, Ozhan Cetindag $^{1}$, Mutlu Unver ${ }^{1}$, Melek Uncel ${ }^{2}$, Burcin Kibar Ozturk ${ }^{3}$, \\ Eyup Kebabci ${ }^{1}$, Umit Bayol ${ }^{2}$ \\ ${ }^{1}$ Tepecik Teaching and Research Hospital, Department of General Surgery, Izmir, Turkey \\ ${ }^{2}$ Tepecik Teaching and Research Hospital, Department of Pathology, Izmir, Turkey \\ ${ }^{3}$ Ege University Faculty of Medicine, Department of Radiology, Izmir, Turkey
}

Email address:

surgeon0052@gmail.com (Safak O.),ozhancetindag@gmail.com (Ozhan C.), mutluunver@gmail.com (Mutlu U.), melek.uncel@gmail.com (Melek U.), burcin_kib@hotmail.com (Burcin K. O.), eyupkebabci@gmail.com (Eyup K.), ubayol@yahoo.com (Umit B.)

\section{To cite this article:}

Safak Ozturk, Ozhan Cetindag, Mutlu Unver, Melek Uncel, Burcin Kibar Ozturk, Eyup Kebabci, Umit Bayol. Mesenteric Fibromyoma Mimicking a Colon Tumor: A Diagnostic Dilemma. Journal of Surgery. Vol. 2, No. 6, 2014, pp. 88-92. doi: 10.11648/j.js.20140206.12

\begin{abstract}
Introduction: Solid primary tumors of mesenteric origin are quite rare among the intraabdominal soft tissue tumors. The most frequent tumors are Gastrointestinal Stromal Tumors (GIST) and smooth muscle tumors. GISTs as the largest group of these tumors are usually seen in the wall of gastrointestinal tract and $25 \%$ of them are malignant. They usually occur in adults and there is no sexual predominance. We present a huge intra-abdominal myoma clinically mimicking GIST. Case Report: A 49-years old male was referred to our deparment because of a growing mass and pain in the right lower quadrant. Physical examination revealed a huge mass with $10 \mathrm{~cm}$ in diameter in the right lower quadrant. Computed tomography (CT) revealed a mass with $17 \mathrm{~cm}$ in diameter which originated from caecum and extented from umblicus to urinary bladder and right colon. The mass was resected en-bloc with right hemicolectomy. The final diagnosis is "Fibromyoma of the mesentery". Discussion: They usually arise de novo and are seen in adults. Myofibroma can occur as a solitary form or it can be seen as multiple lesions like generalized form which is described as myofibromatozis. Myofibromas are characteristically circumscribed masses with a nodular or multinodular growth pattern and they usually show a biphasic growth pattern . The behaviour of mesenteric smooth muscle tumours is unpredictable. Because they arise large diameters and they can cause different clinical symptoms like obstruction and abdominal pain. The treatment of mesenteric fibromyoma is complete resection. Large intraabdominal solid masses pose a diagnostic challenge either grossly or microscopically. Histopathologically, huge benign fibromyomas should be kept in mind of differantial diagnosis of GISTs.
\end{abstract}

Keywords: Colectomy, Gastrointestinal Stromal Tumors, Myofibroma, Smooth Muscle Actin

\section{Introduction}

Solid primary tumors of mesenteric origin are quite rare among the intraabdominal soft tissue tumors [1]. The most frequent tumors are Gastrointestinal Stromal Tumors (GIST) and smooth muscle tumors. GISTs as the largest group of these tumors are usually seen in the wall of gastrointestinal tract and $25 \%$ of them are malignant [2]. They usually occur in adults and there is no sexual predominance. It may also cause intestinal obstruction. Fibromyoma is a benign mesenchymal tumor that can occur at visceral organs such as the heart, lung, liver, kidney, pancreas, gastrointestinal tract and central nervous system $[2,3]$. As well as smooth muscle tumors may mimic GISTs because of their large sizes, their behaviour is entirely based on their histological features by contrast with GISTs [2]. We present a huge intra-abdominal myoma clinically mimicking GIST.

\section{Case Report}

A 49-years old male was referred to our deparment because of a giant intra-abdominal mass and pain in the right lower quadrant. There is no significant anamnesis of trauma and co-morbidites. Physical examination revealed a big mass with $10 \mathrm{~cm}$ in diameter in the right lower quadrant. Laboratory results were within normal limits. Computed 
tomography (CT) revealed a mass with $17 \mathrm{~cm}$ in diameter which originates from caecum and extended from umbilicus to urinary bladder and right colon (Figure 1). A wide median incision was performed and presence of a solid mass probably originating from caecum with $17 \mathrm{~cm}$ in diameter and invading the terminal ileum, appendix and right colon was confirmed intraoperatively. The mass was en-bloc resected with right colectomy and an ileocolic isoperistaltic anastomosis was performed manually with 3/0 PDS sutures (Figure 2). The mean operation time was 150 minutes and the mean blood loss was $300 \mathrm{cc}$. The patient was discharged on the 7 th postoperative day and there is no recurrence in 3th month follow up period. Grossly a $18 \mathrm{~cm}$ diametered solid large lobulated serosal mass is seen at ceacal region. On the cut surface, the gastrointestinal mucosa was invaginated into the mass (Figure 3). The tumor is seen as solid firm tan-gray well-circumscribed. There is no mucosal ulceration. Light microscopy showed that the tumor consisted of loosely and haphazardly arranged spindle cells with bland oval nuclei and the tumor cells are uniformly and organised as anostomosing fascicles (Figure 4). Mitotic activity rate was low and it ranged from 1 to 2 mitoses per 50 high power fields in the hypercellular areas. There is no necrosis, hemorrhagy and atypical mitosis. Immunohistochemically Vimentin and Smooth Muscle Actin were diffusely positive (Figure 5). Expression of CD117, CD34 and S100 were ever found at the cells that making up the tumor. The final diagnosis is '"Fibromyoma of the mesentery".

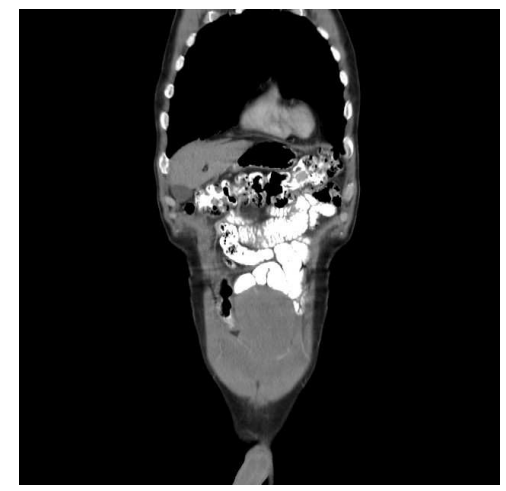

Figure 1.A). The coronal CT image of huge abdominal mass which filled the pelvic area

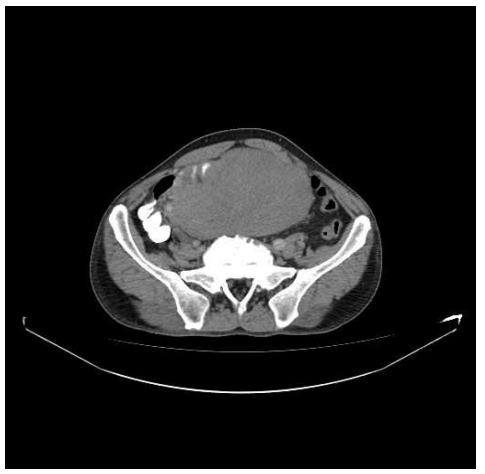

Figure 1.B). The axial CT image of huge abdominal mass which filled the pelvic area

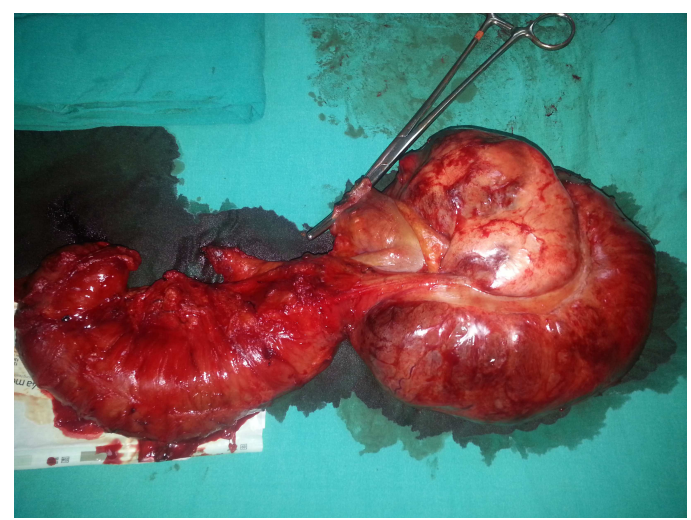

Figure 2. The operative image of en-bloc resected right hemicolectomy specimen

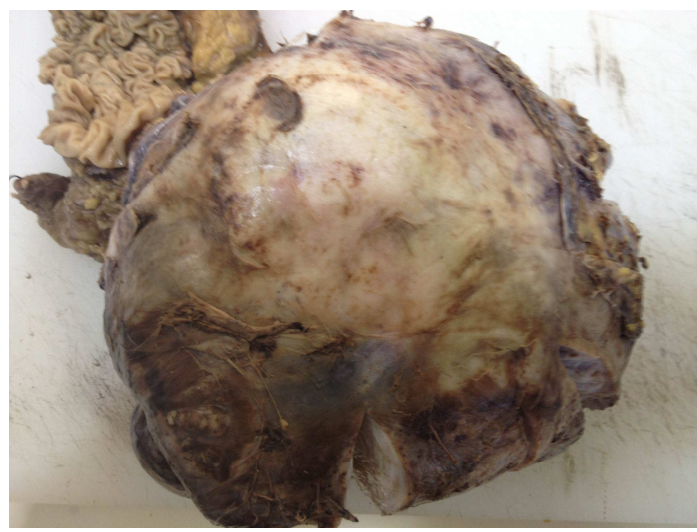

Figure 3.A). External surface of the tumoral mass is covered by brillant, intact serosa.

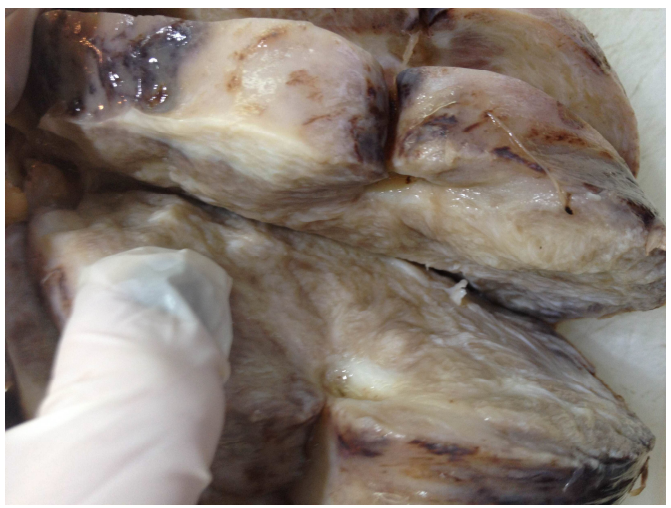

Figure 3.B). Cut surface of the mass is white, fibrous

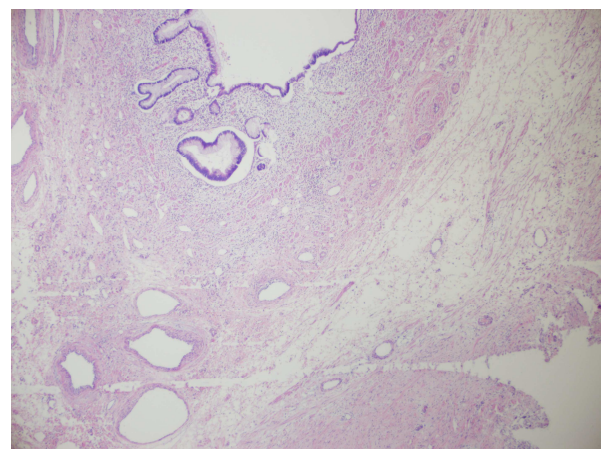

Figure 4.A). Fibromyoma entrapping the original cecal mucosa (H\&E) 


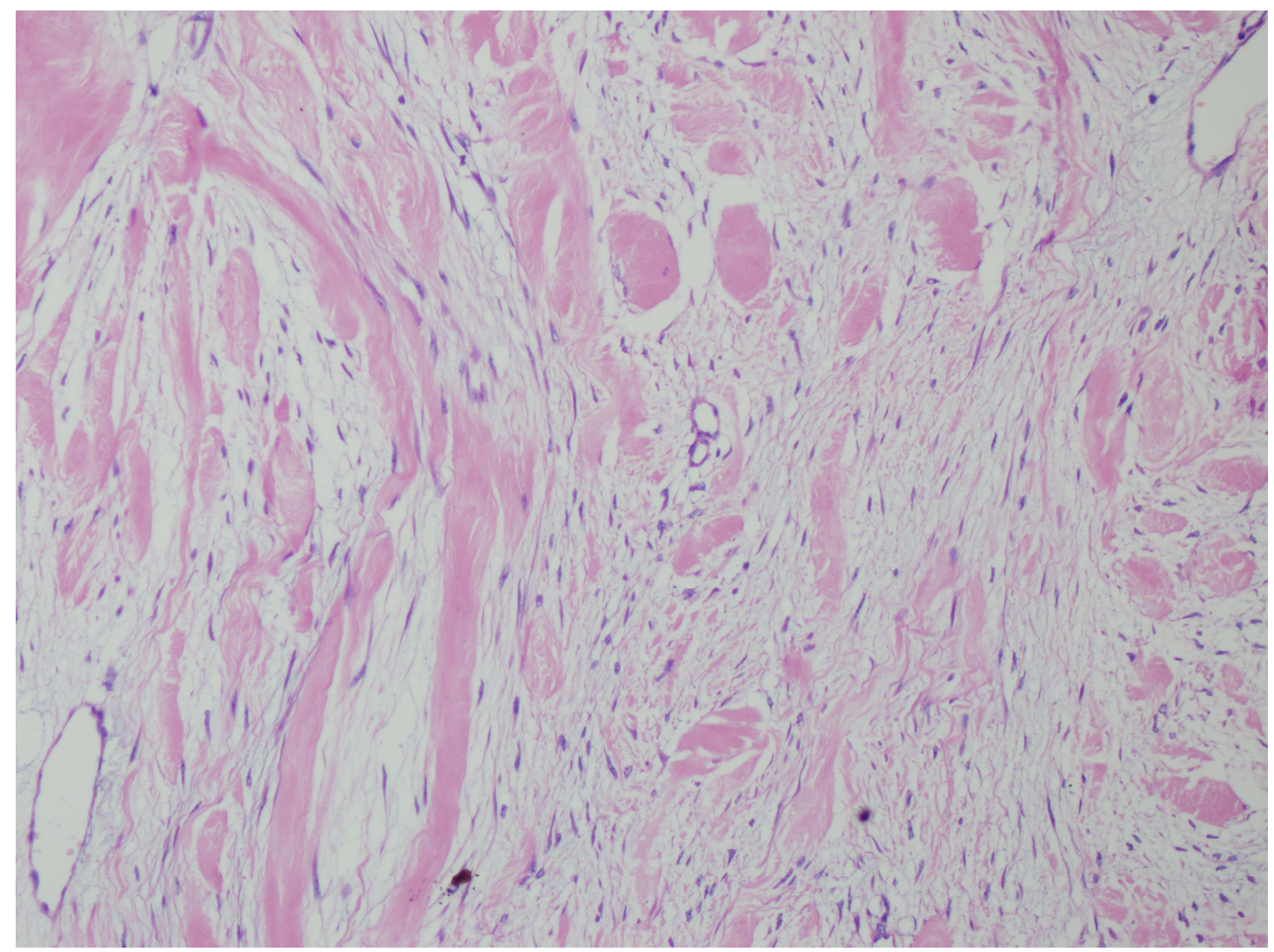

Figure 4.B). Fibromyoma composing of thick collagen bundels and thin fibroblastic cellulary component (H\&E)

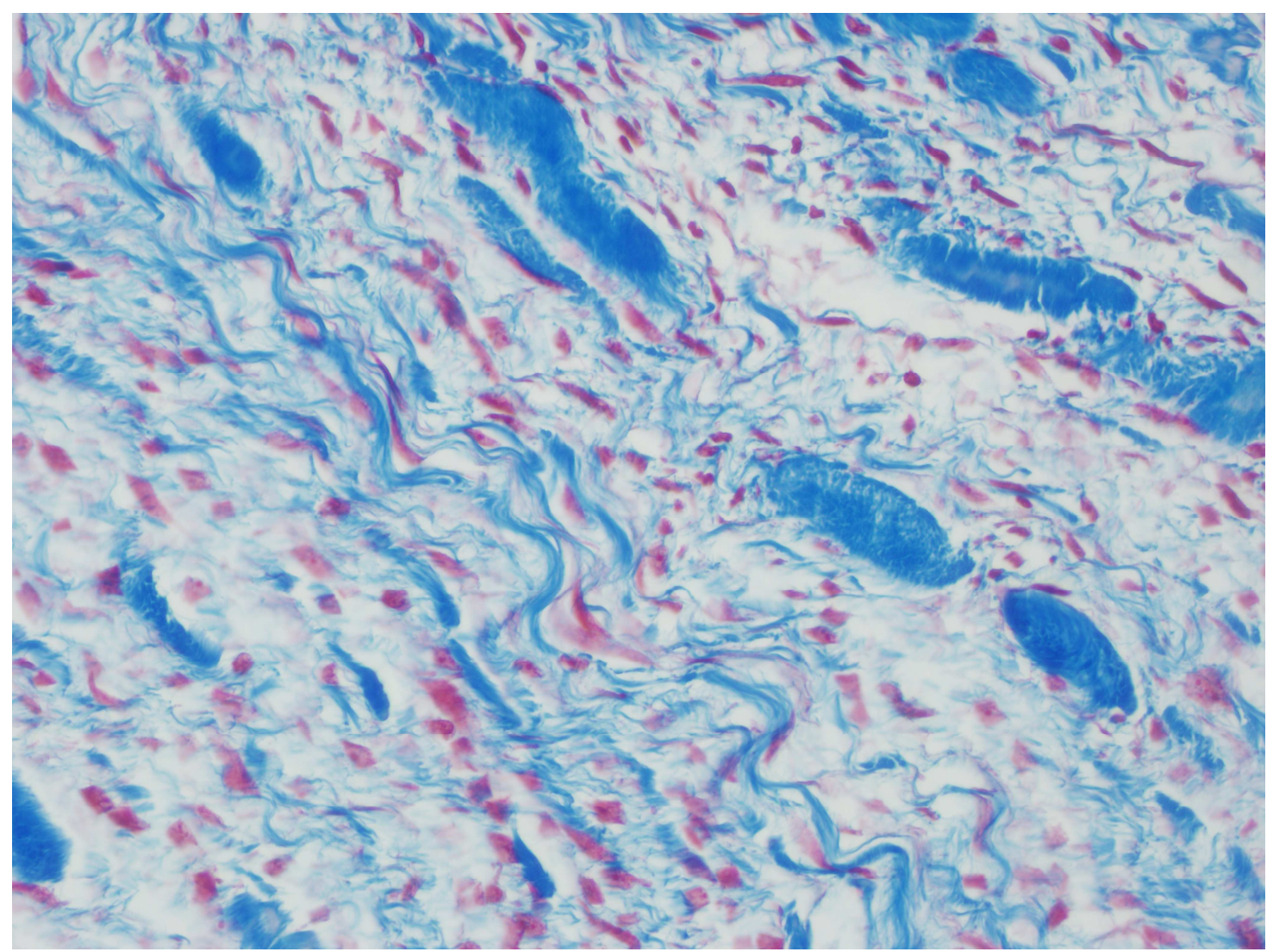

Figure 5.A). Fibromyoma composing of thick collagen bundels and thin fibroblastic cellulary component (Masson's Trichrome) 


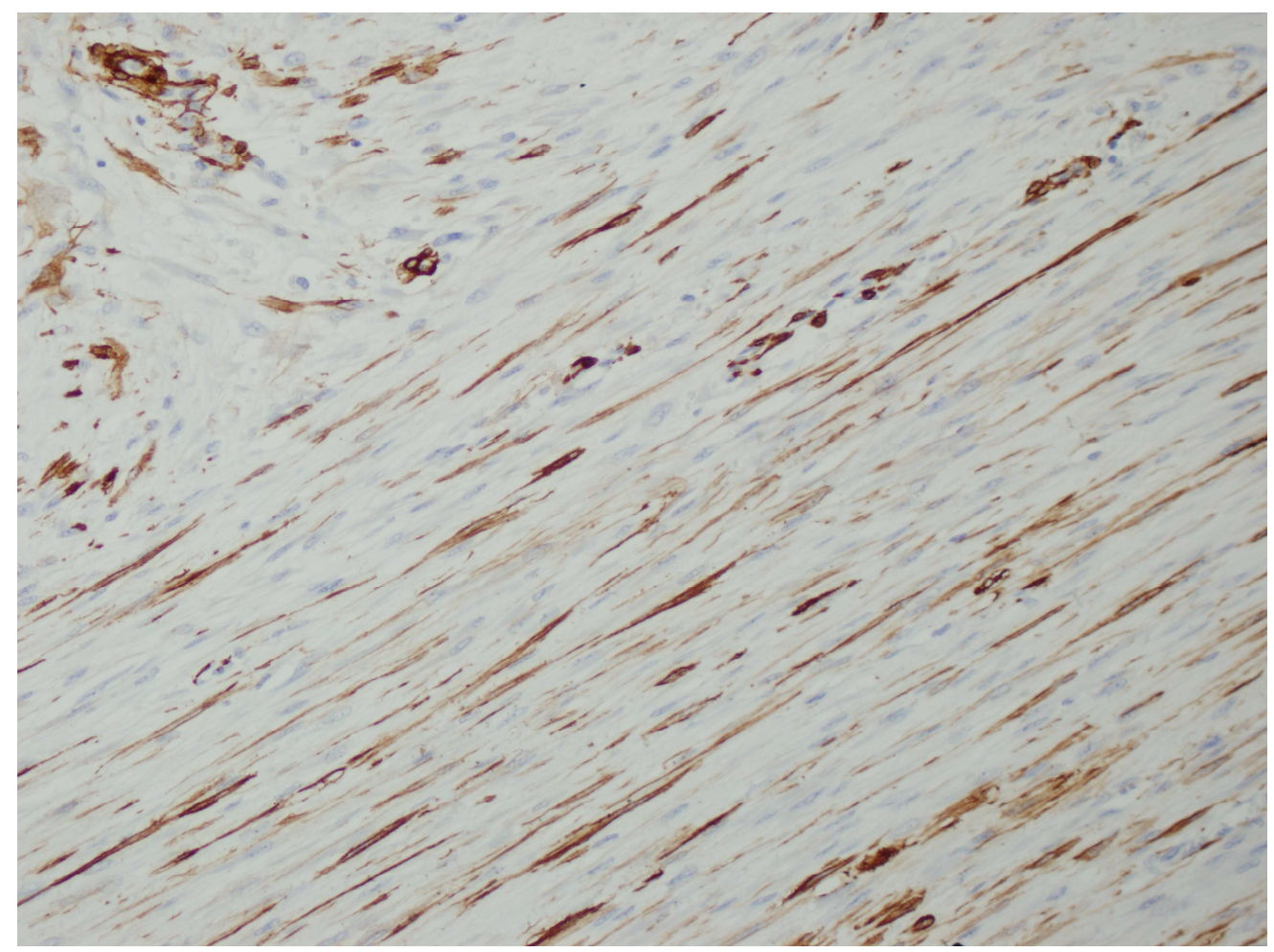

Figure 5.B). Tumor cells reacting positively with actin (Smooth muscle actin)

\section{Discussion}

Solid primary tumors of the mesentery are rare neoplasms among intraabdominal soft tissue tumors and commonly these tumors are smooth muscle tumors and GISTs [1]. Many tumors are also seen in this location including fibromatosis, liposarcoma, malignant fibrous histiocytoma, and peripheral nerve sheath tumors [1, 3-5]. Primary solid mesenteric tumors consist of histological heterogenous group of neoplasms [1]. They usually arise de novo and are seen in adults [6]. Myofibroma can occur as a solitary form or it can be seen as multiple lesions like generalized form which is described as myofibromatozis [2, 7]. It is originally described as 'congenital generalized fibromyomatosis' in 1954 by Stout $[2,8]$. Myofibromas are characteristically circumscribed masses with a nodular or multinodular growth pattern and they usually show a biphasic growth pattern .Consisting of fascicles or whorls of myoid-appearing spindle cells and primitive cells, arranged in a hemangiopericytoma-like pattern $[2,9]$. Most commonly, they are comprised of smooth muscle tumors and GISTs. The behaviour of mesenteric smooth muscle tumours seems to be unpredictable. Because when they arise large diameters, they usually behave like a malignant tumor even in the absence of nuclear atypia, tumor cell necrosis, or increased mitotic count $[1,10]$. In the differential diagnosis, parasitic leiomyoma and benign metastasizing leiomyoma must be keep in mind [11, 12]. In parasitic leiomyoma, the patient had a synchronous uterine leiomyoma and a second detached leiomyoma and attached to the mesentery [11]. In the benign metastasizing leiomyoma, the patient had lesions in the lungs and inadequately excised smooth muscle tumor in the past [12]. The World Health Organization (WHO), in the 2002 classification of soft tissue tumors, recognized myofibromatosis under the benign category of fibroblastic-myofibroblastic lesions [13] . The treatment of mesenteric fibromyoma is complete resection of the lesion. The prognosis is based on the solitary involvement and complete resection of the lesion [2] .

\section{Conclusion}

Solitary myofibromas involving the gastrointestinal tract are extremely rare in adult patients. Solitary myofibroma should be considered in the differential diagnosis of spindle cell tumors of the intestine. The differential diagnosis to distinguish it from malignant tumors is needed. Large intraabdominal solid masses pose a diagnostic challenge either grossly or microscopically. Histopathologically, huge benign fibromyomas should be kept in mind of differantial diagnosis of GISTs. The optimal treatment of mesenteric fibromyoma is complete resection of the lesion.

\section{Consent}

All the authors should confirm that the patient has given their informed consent for the case report to be published.

\section{Acknowledgement}

All the authors declared that the case has been presented as a poster presentation at the $26^{\text {th }}$ European Congress of Pathology. 


\section{References}

[1] Kalogiannidis I, Stavrakis T, Amplianitis I, Grammenou S, Mavromatidis G, Rousso D. Primary Mesenteric Smooth Muscle Tumor: An Entity with Unpredictable Biologic Behavior. Case Rep Obstet Gynecol. 2013; 2013: 483689.

[2] Kim MJ, Lee SH, Youk EG, Lee S, Choi JH, Cho KJ. Solitary myofibroma of the sigmoid colon: case report and review of the literature. Diagn Pathol. 2013; 8: 90.

[3] Colombo P, Rahal D, Grizzi F, Quagliuolo V, Roncalli M. Localized intra-abdominal fibromatosis of the small bowel mimicking a gastrointestinal stromal tumor: a case report. World J Gastroenterol. 2005; 11(33): 5226-8.

[4] Montagliani L, Duverger V. Desmoid Tumors. Journal de Chirurgie. 2008; 67(11): 20-26.

[5] Singla SL, Rattan KN, Kaushik N. Mesenteric Leiomyoma in infancy. Indian Journal of Pediatrics. 2000; 67(11): 857-858.

[6] Estes WL. X. Myofibroma of the Large Intestine. Ann Surg. 1906; 44(2): 249-260.
[7] Chung EB, Enzinger FM. Infantile myofibromatosis. Cancer. 1981; 8: 1807-1818.

[8] Stout AP. Juvenile fibromatoses. Cancer. 1954; 8: 953978.

[9] Fletcher CDM, Unni KK, Mertens F. World health organization classifiction of tumors. Pathology and genetics of tumors of soft tissue and bone. Lyon: IARC Press; 2002.

[10] Yannopoulos K, Stout AP. Primary solid tumors of the mesentery. Cancer. 1963; 16: 914-927.

[11] Fasih N, Shanbhogue AKP, Macdonald DB, et al. Leiomyomas beyond the uterus: unusual locations, rare manifestations. Radiographics. 2008; 28(7): 1931-1948.

[12] Tietze L, Günther K, Hörbe A, et al. Benign metastasizing leiomyoma: a cytogenetically balanced but clonal disease. Human Pathology. 2000; 31(1): 126-128.

[13] Wu W, Chen J, Cao X, Yang M, Zhu J, Zhao G. Solitary infantile myofibromatosis in the bones of the upper extremities: Two rare cases and a review of the literature. Oncology Letter. 2013; 6 (5): 1406-1408. 